\title{
PRIMITIVE INVERSE CONGRUENCES ON CATEGORICAL SEMIGROUPS
}

\author{
JOHN FOUNTAIN ${ }^{1}$ AND GRACINDA M. S. GOMES ${ }^{2}$ \\ ${ }^{1}$ Department of Mathematics, University of York, Heslington, York YO1 5DD, UK \\ ${ }^{2}$ Departamento de Matemática, Faculdade de Ciências, Universidade de Lisboa, \\ 1749-016, Lisboa, Portugal and \\ Centro de Álgebra da Universidade de Lisboa, Av. Prof. Gama Pinto 2, \\ 1649-003 Lisboa, Portugal
}

(Received 27 August 1998)

\begin{abstract}
We give an abstract description of the kernel of a proper primitive inverse congruence on a categorical semigroup. More specifically, we show that it is a $*$-reflexive, $*$-unitary, $*$-dense subsemigroup, and that on a given categorical semigroup there is a one-one correspondence between such subsemigroups and the proper primitive inverse congruences. Our results allow us to give a description of the minimum proper primitive inverse semigroup congruence on a strongly $E^{*}$-dense categorical semigroup.
\end{abstract}

Keywords: congruence; inverse semigroup; primitive idempotents

AMS 1991 Mathematics subject classification: Primary 20M18

\section{Introduction}

It is argued in [10] that when considering semigroups with zero, a case can be made for regarding the class of Brandt semigroups as the natural analogue of the class of groups. Another possible analogue is the related class of primitive inverse semigroups (with zero). The point is that in either class, any semigroup without zero-divisors is a group with an adjoined zero. These thoughts suggest that for semigroups with zero, Brandt semigroup congruences or primitive inverse semigroup congruences might play the role played by group congruences for general semigroups.

Three different, although necessarily equivalent, characterizations of the kernel of a group congruence have been given by Dubreil $[\mathbf{3}]$, Levi $[\mathbf{1 5}, \mathbf{1 6}]$ and Gomes $[\mathbf{8}]$. An account of the approach in [3] and a brief description of that in [15] and [16] is given in [2, ch. 10]. The properties used in the characterizations can be adapted to the case of semigroups with zero. However, it is not clear that the adaptations of the three characterizations are equivalent and the appropriate one for semigroups with zero seems to be the approach of Gomes in [8].

After presenting the basic definitions in $\S 2$, we start $\S 3$ by showing how to associate a proper primitive inverse congruence with a strongly $*$-dense subsemigroup of a categorical 
semigroup. We then investigate the kernel of a proper primitive inverse congruence on a categorical semigroup and show that it is $*$-reflexive, $*$-unitary and $*$-dense. Conversely, any subsemigroup of a categorical semigroup which has these properties is the kernel of a proper primitive inverse congruence.

The question arises of when a semigroup with zero has a proper primitive inverse congruence. Preston [21], extending an earlier result of Munn [19], has shown that the semigroup must be categorical. A categorical inverse semigroup has a proper primitive inverse congruence, but categoricity is not sufficient in the general case. We conclude $\S 3$ with a result suggested by the referee that gives necessary and sufficient conditions for the existence of a proper primitive inverse congruence on an arbitrary semigroup.

We consider $E^{*}$-dense semigroups in $\S 4$ and give a description of the minimum proper primitive inverse congruence on a strongly $E^{*}$-dense categorical semigroup, which is an analogue of the description in [18] of the minimum group congruence on an $E$-dense semigroup.

In $\S 5$, we specialize to the case of categorical inverse semigroups and examine the connections between our approach and the kernel-trace approach of Petrich [20, ch. III]. Finally, in $\S 6$, we give some analogues of results due to Edwards [4].

\section{Preliminaries}

For the standard terminology and notation of semigroup theory we refer the reader to [12]. In particular, $E(S)$ denotes the set of idempotents of a semigroup $S$, and a subsemigroup $T$ of $S$ is full if $E(S) \subseteq T$. We adopt the usual convention that a semigroup with zero must contain at least two elements. By a proper congruence on a semigroup with zero we mean a congruence $\rho$ such that $0 \rho=\{0\}$. Proper congruences are also widely known as 0-restricted congruences (see, for example, [2]). We occasionally use the term 0-subsemigroup to emphasize that 0 is a member of the subsemigroup under consideration. The 0 -subsemigroup generated by a subset $A$ of a semigroup with zero will be denoted by $\langle A\rangle^{0}$.

Many concepts of semigroup theory can be modified to give closely related ideas which are more useful in the context of semigroups with zero. We now describe how the notions of dense, reflexive and unitary subsets of a semigroup are modified. Let $T$ be a subset of a semigroup $S$ with zero. The set of non-zero elements of $T$ is denoted by $T^{*}$; in particular, $E^{*}(S)$ or just $E^{*}$ is the set of non-zero idempotents of $S$.

We say that $T$ is $*$-dense in $S$ if for every non-zero element $a$ of $S$ there are elements $x, y \in S$ such that $a x, y a \in T^{*}$.

We define $T$ to be $*$-reflexive if $a b \in T^{*}$ implies that $b a \in T^{*}$ for all $a, b \in S$.

Next, $T$ is said to be $*$-unitary if for all elements $a$ of $S$ and $t$ of $T$ we have $a \in T$ if at or $t a$ is in $T^{*}$.

For an element $a$ of $S$, define the sets $X_{T}(a)$ and $Y_{T}(a)$ by

$$
\begin{aligned}
X_{T}(a) & =\left\{b \in S \mid a t b \in T^{*} \text { for all } t \in T^{1} \text { such that } a t \neq 0 \text { or } t b \neq 0\right\}, \\
Y_{T}(a) & =\left\{b \in S \mid b t a \in T^{*} \text { for all } t \in T^{1} \text { such that } t a \neq 0 \text { or } b t \neq 0\right\} .
\end{aligned}
$$

Note that, in particular, if $b \in X_{T}(a)$, then $a b \in T^{*}$, and, if $b \in Y_{T}(a)$, then $b a \in T^{*}$. 
Now put $W_{T}^{*}(a)=X_{T}(a) \cap Y_{T}(a)$.

We say that $T$ is strongly *-dense if $T$ has no non-zero nilpotent elements and $W_{T}^{*}(a)$ is non-empty for all non-zero elements $a$ of $S$. Note that, by definition, a strongly *-dense subsemigroup is $*$-dense. Indeed, it satisfies the condition that for every non-zero element $a$ of $S$ there is an element $b$ such that $a b, b a \in T^{*}$.

The following two lemmas are immediate from the definitions and will be used frequently without further mention.

Lemma 2.1. Let $T$ be a *-reflexive subset of a semigroup $S$ with zero. Then the following conditions are equivalent:

(1) $T$ is *-unitary;

(2) for all elements $a$ of $S$ and $t$ of $T$, if at $\in T^{*}$, then $a \in T$; and

(3) for all elements $a$ of $S$ and $t$ of $T$, if $t a \in T^{*}$, then $a \in T$.

Lemma 2.2. Let $T$ be a *-reflexive subset of a semigroup $S$ with zero. Then the following conditions are equivalent:

(1) $T$ is *-dense;

(2) for every non-zero element $a$ of $S$, there is an element $x$ of $S$ such that ax $\in T^{*}$;

(3) for every non-zero element $a$ of $S$, there is an element $y$ of $S$ such that $y a \in T^{*}$; and

(4) for every non-zero element $a$ of $S$, there is an element $z$ of $S$ such that az $\in T^{*}$ and $z a \in T^{*}$.

A semigroup $S$ is categorical if it has a zero, and, for elements $a, b$ and $c$ of $S$ such that $a b c=0$, we have $a b=0$ or $b c=0$. Categorical semigroups are also said to be categorical at zero (see [2]). Note that when we say that a semigroup is categorical, it is implicit that the semigroup has a zero.

Lemma 2.3. Let $S$ be a categorical semigroup and $T$ be a *-reflexive subsemigroup of $S$. Let $a, b$ be elements of $S$ with $a b \in T^{*}$. If $t \in T$ and $a t \neq 0$ or $t b \neq 0$, then $a t b \in T^{*}$.

Proof. Let $a, b \in S$ and $t \in T$ be such that $a b \in T^{*}$ and $a t \neq 0$. Then $b a \in T^{*}$ since $T$ is *-reflexive, and bat $\neq 0$ since $S$ is categorical. Hence, bat $\in T^{*}$ and, consequently, $a t b \in T^{*}$ as required.

Similarly, if $t b \neq 0$, then we also have $a t b \in T^{*}$.

Corollary 2.4. If $T$ is a *-reflexive, *-dense subsemigroup of a categorical semigroup $S$, then $T$ is strongly *-dense.

Proof. Since $T$ is *-reflexive and $*$-dense, if $a$ is a non-zero element of $S$, there is an element $z$ such that $a z, z a \in T^{*}$. It is now immediate from the lemma that $z \in W_{T}^{*}(a)$.

If $t \in T^{*}$, then, by *-reflexivity and *-denseness, $t y, y t \in T^{*}$ for some $y \in S$. By categoricity, tyt $\in T^{*}$ and so $y t^{2} \in T^{*}$. Hence, $t^{2} \neq 0$. It follows that $T$ is strongly *-dense. 
A Brandt semigroup is an inverse completely 0-simple semigroup. An inverse semigroup $S$ with zero is said to be primitive if every non-zero idempotent $e$ in $S$ is primitive, that is, for all $f \in E^{*}(S)$, if $e \leqslant f$, then $e=f$. For instance, Brandt semigroups are primitive, and, in fact, every primitive inverse semigroup is a 0 -direct union of Brandt semigroups (see, for example, [20, Theorem III.4.3] or [22, Corollary 2]). From [2, Lemma 7.61] we have the following result.

Lemma 2.5. A primitive inverse semigroup is categorical.

The following lemma is a special case of [2, Lemma 7.63(i)].

Lemma 2.6. Let $S$ be a primitive inverse semigroup. Then, for $e \in E(S)$ and $s \in S$, if es $\neq 0$, then $e s=s$. Similarly, se $\neq 0$ implies $s e=s$.

A proper congruence $\rho$ on a semigroup $S$ with zero is said to be a primitive inverse (Brandt) congruence if $S / \rho$ is a primitive inverse (Brandt) semigroup. As we are concerned only with proper congruences, when we use the phrases 'primitive inverse congruence' or 'Brandt congruence' it is implicit that the congruence is proper.

By the kernel of a congruence $\rho$ on a semigroup $S$ we mean the subset $\operatorname{Ker} \rho$ of $S$ defined by

$$
\operatorname{Ker} \rho=\{a \in S \mid a \rho \in E(S / \rho)\}=\left\{a \in S \mid a \rho a^{2}\right\} .
$$

Observe that if $\rho$ is a proper congruence and $a$ is a non-zero element of $\operatorname{Ker} \rho$, then $a^{2} \neq 0$ and, consequently, Ker $\rho$ has no non-zero nilpotent elements.

\section{The characterization}

Throughout this section $S$ will be a categorical semigroup. We re-emphasize that saying a semigroup is categorical implies that it has a zero. Our main aim is to characterize the kernels of proper primitive inverse congruences on $S$ as the *-unitary, *-reflexive, *-dense subsemigroups of $S$. We then show that there is an order isomorphism between the set of all such subsemigroups of $S$ and the set of all proper primitive inverse congruences on $S$.

First, given any strongly *-dense subsemigroup $T$ of $S$ we define a congruence $\rho_{T}$ on $S$ as follows. For all $a, b \in S$,

$$
(a, b) \in \rho_{T} \text { if and only if } a=b=0 \text { or } x a=b t \neq 0 \text { for some } x, t \in T \text {. }
$$

The following proposition is an analogue of [7, Corollary 3.2].

Proposition 3.1. Let $T$ be a strongly *-dense subsemigroup of $S$. Then the relation $\rho_{T}$ is a primitive inverse congruence on $S$ and $T \subseteq \operatorname{Ker} \rho_{T}$.

Proof. Note that if $\rho_{T}$ is a congruence, then, by definition, it is a proper congruence.

For any non-zero element $a$ of $S$ there is an element $x$ such that $a x, x a \in T^{*}$ since $T$ is strongly *-dense. Now $S$ is categorical so that axa $\neq 0$ and, hence, $(a, a) \in \rho_{T}$ and $\rho_{T}$ is reflexive. 
Now let $a$ and $b$ be non-zero elements of $S$ such that $(a, b) \in \rho_{T}$. Then there are elements $x, t$ of $T$ such that $x a=b t \neq 0$. Let $a^{\prime} \in W_{T}^{*}(a), b^{\prime} \in W_{T}^{*}(b)$. Since $x \in T$ and $x a \neq 0$, we have $a^{\prime} b t=a^{\prime} x a \in T^{*}$ and, similarly, $x a b^{\prime}=b t b^{\prime} \in T^{*}$. Now, by categoricity,

$$
a\left(a^{\prime} x a\right)\left(b^{\prime} b\right)=\left(a a^{\prime}\right)\left(b t b^{\prime}\right) b \neq 0
$$

and $\left(a^{\prime} x a\right)\left(b^{\prime} b\right),\left(a^{\prime} a\right)\left(b t b^{\prime}\right) \in T$ so that $(b, a) \in \rho_{T}$ and $\rho_{T}$ is symmetric.

To prove that $\rho_{T}$ is transitive, let $a, b, c \in S^{*}$ be such that $(a, b),(b, c) \in \rho_{T}$. Let $x, t, u, v \in T$ be such that

$$
x a=b t \neq 0 \quad \text { and } \quad u b=c v \neq 0
$$

Then $u b t \neq 0$ and so $u x a=u b t=c v t \neq 0$, where $u t, v t \in T$. Thus $(a, c) \in \rho_{T}$.

Next, we show that $\rho_{T}$ is right compatible. Let $a, b, c \in S^{*}$ and suppose that $(a, b) \in \rho_{T}$. Then $x a=b t \neq 0$ for some $x, t \in T$. Clearly, if $a c=b c=0$, then $(a c, b c) \in \rho_{T}$.

If $b c \neq 0$, let $b^{\prime} \in W_{T}^{*}(b)$ and $c^{\prime} \in W_{T}^{*}(c)$. Since $b^{\prime} b \neq 0$ we have $b^{\prime} x a=b^{\prime} b t \neq 0$ and so $b^{\prime} x \neq 0$. Also, $b c c^{\prime} \neq 0$ by categoricity, so that $b c c^{\prime} b^{\prime} \in T^{*}$ and, hence, $b c c^{\prime} b^{\prime} x \in T^{*}$.

Now $b^{\prime} b \in T^{*}$ and $b^{\prime} b c \neq 0$ by categoricity, so $c^{\prime} b^{\prime} b c \in T^{*}$. It follows that $c^{\prime} b^{\prime} b t \neq 0$ and so $c^{\prime} b^{\prime} b t c \in T^{*}$. In particular, $b t c \neq 0$ so that $x a c \neq 0$. Now, by categoricity,

$$
(b c)\left(c^{\prime} b^{\prime} b t c\right)=\left(b c c^{\prime} b^{\prime} x\right)(a c) \neq 0
$$

so that $(a c, b c) \in \rho_{T}$.

Since $\rho_{T}$ is symmetric, it follows that if $a c \neq 0$, then we also have $(a c, b c) \in \rho_{T}$ and so $\rho_{T}$ is right compatible. A similar argument shows that it is also left compatible.

Next we show that $T \subseteq \operatorname{Ker} \rho_{T}$. Certainly, $0 \in \operatorname{Ker} \rho_{T}$. Let $t$ be a non-zero element of $T$. Then $t^{2} \neq 0$ since $T$ has no nilpotents and so $t^{3} \neq 0$ by categoricity. Hence, $\left(t, t^{2}\right) \in \rho_{T}$ and $t \in \operatorname{Ker} \rho_{T}$.

Now suppose that $\left(a^{2}, a\right) \in \rho_{T}$ for some non-zero element $a$ of $S$. Then $a q \in T^{*}$ for some $q \in S$ and we have $a^{2} q \rho_{T} a q$. Hence, we have $t, x \in T$ such that

$$
a(a q t)=x(a q) \neq 0 \text {. }
$$

Now $a q t, x \in T$ so that $a \rho_{T} a q$ and $a$ is related to an element of $T$. Thus we have shown that

$$
E\left(S / \rho_{T}\right)=\left\{t \rho_{T} \mid t \in T\right\}
$$

We now show that $E\left(S / \rho_{T}\right)$ is a semilattice. If $t, u \in T$ and $t u \neq 0$, then $(t u)^{2} \neq 0$ so that $u t \neq 0$. Thus $u(t u)=(u t) u \neq 0$, so that $(t u, u t) \in \rho_{T}$. If $t u=0$, then clearly $u t=0$, so that, again, $(t u, u t) \in \rho_{T}$. Hence $E\left(S / \rho_{T}\right)$ is a semilattice.

Next we show that $S / \rho_{T}$ is regular and, hence, inverse. If $a$ is a non-zero element of $S$, then $a x, x a \in T^{*}$ for some $x \in S$. Hence $(x a)^{2} \in T$ and

$$
a x(a x a)=a(x a)^{2} \neq 0
$$

so that $(a, a x a) \in \rho_{T}$ and $S / \rho_{T}$ is regular. 
Finally, we show that $S / \rho_{T}$ is primitive. Let $t \rho_{T}, u \rho_{T}$ be non-zero idempotents of $S / \rho_{T}$ and suppose that $t \rho_{T} \leqslant u \rho_{T}$, so that $(t, u t) \in \rho_{T}$. Then $x t=u t y \neq 0$ for some elements $x, y$ of $T$. We can assume that $t, u \in T$ and so we have $(t, u) \in \rho_{T}$ since $t y \in T$.

Therefore, $S / \rho_{T}$ is a primitive inverse semigroup.

Our next result, which corresponds to $[\mathbf{8}$, Lemma 5$]$, shows that every primitive inverse congruence on $S$ is of the form $\rho_{K}$ for some $*$-unitary, *-reflexive, *-dense subsemigroup $K$ of $S$.

Proposition 3.2. Let $\rho$ be a primitive inverse congruence on $S$. Then Ker $\rho$ is a *unitary, *-reflexive, $*$-dense subsemigroup of $S$ and $\rho=\rho_{\text {Ker } \rho}$.

Proof. Let $K=\operatorname{Ker} \rho$. Suppose that $a, b \in S$ and $a b \in K^{*}$. Then $(a \rho)(b \rho)=(a b) \rho$ is a non-zero idempotent of $S / \rho$. Since $S / \rho$ is primitive inverse, $(b a) \rho=(b \rho)(a \rho)$ is also a non-zero idempotent. Thus, $b a \in K^{*}$ and $K$ is *-reflexive.

If $a$ is a non-zero element of $S$, then $a \rho$ is a non-zero element of $S / \rho$ and has an inverse $(a \rho)^{-1}$ in $S / \rho$. Let $(a \rho)^{-1}=x \rho$. Then $(a x) \rho=(a \rho)(x \rho)=(a \rho)(a \rho)^{-1}$ is a nonzero idempotent of $S / \rho$ so that $a x \in K^{*}$. Thus $K$ is *-dense.

If $a, b \in S$ and $a b, b \in K^{*}$, then $(a \rho)(b \rho)$ and $b \rho$ are non-zero idempotents of $S / \rho$. Since all idempotents in $S / \rho$ are primitive, it follows that $a \rho \mathcal{L} b \rho$ and so $(a \rho)(b \rho)=a \rho$. Thus $a \rho$ is a non-zero idempotent. Hence $K$ is $*$-unitary as required.

If $a, b \in S^{*}$ and $(a, b) \in \rho$, then, since $S / \rho$ is inverse, we have $(a \rho)^{-1}=(b \rho)^{-1}=x \rho$ for some non-zero element $x$ of $S$. Now $x a, b x \in K$ and $(b x) a=b(x a) \neq 0$ so that $(a, b) \in \rho_{K}$.

Conversely, if $(a, b) \in \rho_{K}$ and $a, b$ are non-zero, then $u a=b v \neq 0$ for some $u, v \in K$. In $S / \rho$, the element $(u \rho)(a \rho)$ is non-zero and $u \rho \in E(S / \rho)$ so that by Lemma 2.6, $(u \rho)(a \rho)=a \rho$. Similarly, $(b \rho)(v \rho)=b \rho$ so that $(a, b) \in \rho$. Thus $\rho=\rho_{K}$ as required.

Note that a non-zero intersection of *-reflexive, *-unitary subsemigroups of $S$ is again *-reflexive and *-unitary. Let $T$ be a non-zero subsemigroup of $S$ and suppose that $T$ is contained in a $*$-reflexive, $*$-unitary subsemigroup. Let $T_{\infty}$ be the least $*$-reflexive, *-unitary subsemigroup of $S$ containing $T$. Note that if $T$ is $*$-dense, then $T_{\infty}$ is also *-dense since $T \subseteq T_{\infty}$.

We can construct $T_{\infty}$ from $T$ in the following way. First, let $A$ be a non-zero 0subsemigroup of $S$ that is contained in a $*$-reflexive, $*$-unitary 0 -subsemigroup $B$. Let

$$
u^{*}(A)=\left\{x \in S \mid x y \text { or } y x \text { is in } A^{*} \text { for some } y \in A^{1}\right\} .
$$

Since $B$ is *-unitary, we have $A^{*} \subseteq u^{*}(A) \subseteq B$ and so the 0-subsemigroup $\left\langle u^{*}(A)\right\rangle^{0}$ generated by $u^{*}(A)$ is contained in $B$. Let

$$
r^{*}(A)=\left\{x \in S \mid x=y z \text { for some } y, z \in S^{1} \text { such that } z y \in A^{*}\right\} .
$$

Note that if $x \in r^{*}(A)$, then $x \neq 0$. This is immediate if $y=1$ or $z=1$. If $y, z \in S$, then $z y \in B^{*}$ so that $y z \in B^{*}$ and $y z \neq 0$. Observe also that since $B$ is *-reflexive, $A \subseteq\left\langle r^{*}(A)\right\rangle^{0} \subseteq B$. 
Now let $T_{0}=T$ and for $k \geqslant 0$, let $T_{2 k+1}=\left\langle u^{*}\left(T_{2 k}\right)\right\rangle^{0}$ and $T_{2 k+2}=\left\langle r^{*}\left(T_{2 k+1}\right)\right\rangle^{0}$. Then we have an ascending chain

$$
T_{0} \subseteq T_{1} \subseteq T_{2} \subseteq \cdots
$$

and $T^{\prime}=\bigcup_{k \geqslant 0} T_{k}$ is a subsemigroup of $S$. Since $T_{0} \subseteq T_{\infty}$, it follows by induction and the above remarks that $T_{k} \subseteq T_{\infty}$ for all $k$. Thus $T^{\prime} \subseteq T_{\infty}$.

If $a b \in T^{\prime}$ and $a b \neq 0$, then $a b \in T_{2 k+1}^{*}$ for some $k$. Thus $b a \in r^{*}\left(T_{2 k+1}\right)$ so that $b a$ is a non-zero element of $T^{\prime}$ and, hence, $T^{\prime}$ is *-reflexive.

If $a b, b$ are non-zero elements of $T^{\prime}$, then we have $a b, b \in T_{2 k}^{*}$ for some $k$ and so $a \in u^{*}\left(T_{2 k}\right)$. It follows that $T^{\prime}$ is *-unitary and, consequently, $T_{\infty}=T^{\prime}$.

Now we show that if $T$ is a strongly *-dense subsemigroup of $S$, then $T_{\infty}$ is the kernel of the primitive inverse congruence $\rho_{T}$.

Proposition 3.3. If $T$ is a strongly *-dense subsemigroup of $S$, then

$$
T_{\infty}=\operatorname{Ker} \rho_{T} \quad \text { and } \quad \rho_{T}=\rho_{T_{\infty}} .
$$

Proof. By Proposition 3.1, $T$ is contained in $\operatorname{Ker} \rho_{T}$ and, by Proposition 3.2, Ker $\rho_{T}$ is $*$-unitary and *-reflexive. Hence, by definition, $T_{\infty} \subseteq \operatorname{Ker} \rho_{T}$.

On the other hand, if $a \in \operatorname{Ker} \rho_{T}$, then, as in the proof of Proposition 3.1, $(a, t) \in \rho_{T}$ for some $t \in T$. If $a$ is non-zero, then there are elements $x, v$ of $T$ such that $a x=v t \neq 0$. But $v t \in T$ and $T \subseteq T_{\infty}$, so that $a x \in T_{\infty}^{*}$ and, hence, $a \in T_{\infty}^{*}$ since $T_{\infty}$ is $*$-unitary.

Thus $T_{\infty}=\operatorname{Ker} \rho_{T}$, and so, by Proposition $3.2, \rho_{T}=\rho_{T_{\infty}}$.

We can now put our results together to obtain the main theorem of the section.

Theorem 3.4. The mappings $T \mapsto \rho_{T}$ and $\rho \mapsto \operatorname{Ker} \rho$ are mutually inverse order isomorphisms between the set of *-unitary, *-reflexive, *-dense subsemigroups of $S$ and the set of all primitive inverse congruences on $S$.

Proof. If $T$ is a $*$-unitary, *-reflexive, *-dense subsemigroup of $S$, then $\rho_{T}$ is a primitive inverse congruence by Proposition 3.1. By Proposition 3.3, $\operatorname{Ker} \rho_{T}=T_{\infty}$ and $T=T_{\infty}$ since $T$ is *-unitary, *-reflexive and $*$-dense.

On the other hand, if $\rho$ is a primitive inverse congruence on $S$, then $\operatorname{Ker} \rho$ is $*$-unitary, $*$-reflexive and $*$-dense, and $\rho=\rho_{\text {Ker } \rho}$ by Proposition 3.2.

Thus the two mappings are mutually inverse. It is straightforward to verify that they are order-preserving.

An immediate consequence of the theorem is the following corollary.

- Corollary 3.5. Let $\rho, \theta$ be primitive inverse congruences on $S$. Then $\rho=\theta$ if and only if $\operatorname{Ker} \rho=\operatorname{Ker} \theta$.

We now turn our attention to Brandt congruences. We note first that if a semigroup $R$ has a Brandt congruence, then, by [19, Theorem 1.1], $R$ is categorical, and also any two non-zero ideals of $R$ have non-zero intersection. Following [9], we say that such a semigroup is strongly categorical. 
It is not difficult to show that if $R$ is a strongly categorical semigroup and if $\tau$ is any proper congruence on $R$, then $R / \tau$ is strongly categorical. Hence, if $\rho$ is a primitive inverse congruence on $R$, then $R / \rho$ is a Brandt semigroup. The following corollary is, therefore, an immediate consequence of Theorem 3.4.

Corollary 3.6. Let $R$ be a strongly categorical semigroup. Then the mappings $T \mapsto$ $\rho_{T}$ and $\rho \mapsto \operatorname{Ker} \rho$ are mutually inverse order isomorphisms between the set of $*$-unitary, *-reflexive, *-dense subsemigroups of $R$ and the set of all Brandt congruences on $R$.

Having characterized proper primitive inverse congruences by their kernels, we now consider when such congruences exist. We start by quoting the following result $[\mathbf{1 7}$, Proposition 1].

Proposition 3.7. Let $S$ be a semigroup with zero. Then

$$
\rho=\left\{(a, b) \in S \times S \mid \text { for all } s, t \in S^{1}, \text { sat }=0 \text { if and only if } s b t=0\right\}
$$

is a proper congruence on $S$. If $\tau$ is any proper congruence on $S$, then $\tau \subseteq \rho$.

Borrowing terminology from ring theory, we say that a semigroup $S$ with zero is semiprime if $a S a \neq 0$ for every non-zero element $a$ of $S$. A homomorphism $\alpha: S \rightarrow T$ of semigroups with zero is proper if $0 \alpha^{-1}=\{0\}$; that is, $\alpha$ is proper if and only if the congruence it induces is proper.

Theorem 3.8. Let $S$ be a semigroup with zero and let

$$
U=\left\{a \in S \mid a^{2} \neq 0\right\} \cup\{0\} .
$$

Then the following statements are equivalent:

(1) $S$ has a proper primitive inverse homomorphic image;

(2) $S$ is categorical, semiprime, and, for all $a, b$ in $S$,

$$
a^{2} \neq 0, \quad b^{2} \neq 0, \quad a b \neq 0, \quad \text { together imply that } b a \neq 0 ;
$$

(3) $S$ is categorical and $U$ is a $*$-dense subsemigroup; and

(4) $S$ is categorical and $U$ is a *-unitary, *-reflexive, *-dense subsemigroup.

Proof. Suppose first that $S$ has a proper primitive inverse homomorphic image, and let $\theta$ be a proper homomorphism onto a primitive inverse semigroup. Then, by $[2$, Lemma 7.62], $S$ is categorical. If $a$ is a non-zero element of $S$, then $a \theta \neq 0$, so that, if $b \theta$ is the inverse of $a \theta$, then $a b a \neq 0$ since $\theta$ is proper, and, hence, $S$ is semiprime.

A homomorphic image of an inverse semigroup is inverse [6, Theorem 5.1.4] and it follows easily from Lallement's Lemma [6, Lemma 2.4.4] that any non-zero idempotent in a homomorphic image of a primitive inverse semigroup is primitive. Thus, the class of primitive inverse semigroups is closed under homomorphic images, and so, by [17, Proposition 2], $S / \rho$ is primitive inverse. Now let $a, b \in S$ be such that $a^{2}, b^{2}$ and $a b$ are 
non-zero. By categoricity, it follows that $s a^{2} t \neq 0$ if and only if sat $\neq 0$, and, hence, $a \rho$ is idempotent. Similarly, $b \rho$ is also idempotent and so $(a \rho)(b \rho)=(b \rho)(a \rho)$ since $S / \rho$ is inverse. Thus $b a \neq 0$ and (2) holds.

Now suppose that (2) holds and let $a, b \in U^{*}$ so that $a^{2}$ and $b^{2}$ are non-zero. If $a b \neq 0$, then $b a \neq 0$ by assumption, and so, by categoricity, $a b a b \neq 0$, whence $a b \in U$ and $U$ is a subsemigroup.

Let $s$ be a non-zero element of $S$. Then $s S s \neq 0$ since $S$ is semiprime. Let $b \in S$ be such that $s b s \neq 0$. By categoricity, $s b s b \neq 0$ and $b s b s \neq 0$ so that $s b, b s \in U$ and $U$ is *-dense in $S$.

Next, suppose that (3) holds. If $a b \in U^{*}$, then $(a b)^{2} \neq 0$ so that $b a \neq 0$, and, hence, by categoricity, $(b a)^{2} \neq 0$, that is, $b a \in U^{*}$. Thus $U$ is $*$-reflexive.

If $s \in S, u \in U$ and $s u \in U^{*}$, then $u^{2} \neq 0$ and $(s u)^{2} \neq 0$ so that, by categoricity, $(u s)^{2} \neq 0$, that is, $u s \in U$. Hence, $s u^{2} s \in U$ and, by categoricity again, $s u^{2} s \neq 0$. Thus $\left(s u^{2} s\right)^{2} \neq 0$, so that $s^{2} \neq 0$, that is, $s \in U$ and, therefore, $U$ is $*$-unitary.

Finally, if (4) holds, then, by Corollary 2.4 and Proposition $3.1, \rho_{U}$ is a proper primitive inverse congruence on $S$.

\section{4. $E^{*}$-dense semigroups}

A semigroup $S$ with zero is $E^{*}$-dense if, for every non-zero element $a$ of $S$, there are elements $b, c$ of $S$ such that $a b, c a \in E^{*}(S)$. An $E^{*}$-dense semigroup is obviously $E$-dense and the notion is an analogue for the class of semigroups with zero of the concept of $E$ denseness in the class of all semigroups. An $E$-dense semigroup is said to be $E$-inversive in [2]; $E^{*}$-dense semigroups are called 0 -inversive in [14] and weakly regular in [17].

The analogy between $E$-dense and $E^{*}$-dense semigroups is far from perfect. For example, it is known that every $E$-dense semigroup has a minimum group congruence. The existence of such a congruence was noted in [11] and an explicit description given in [18, Proposition 9]. In contrast, as we have already noted, the work of Munn and Preston shows that the existence of a (Brandt) primitive inverse congruence on a semigroup forces the semigroup to be (strongly) categorical. For an inverse semigroup, Preston (Munn) shows that (strong) categoricity is sufficient, and Fountain and Hayes [6] prove a similar result for an $E^{*}$-dense semigroup in which the idempotents commute with each other. However, we have seen that categoricity by itself is not sufficient for a general semigroup with zero to have a primitive inverse congruence and the same is true for $E^{*}$-dense semigroups.

Let $T$ be a semigroup with zero such that $E(T)$ is a commutative subsemigroup. Then, by [14, Theorem 3], $T$ is primitive inverse if and only if it is $E^{*}$-dense and satisfies the following weak cancellation law:

$$
\text { if } a, b, x, y \in T \text {, then } a x=b x \neq 0 \text { and } y a=y b \neq 0 \text { together imply that } a=b \text {. }
$$

Using this result it is easy to see that if $I$ is the set of proper primitive inverse congruences on an $E^{*}$-dense semigroup $S$, and if $\sigma=\bigcap\{\tau: \tau \in I\}$, then, provided that $I$ is not empty, $\sigma$ is the minimum proper primitive inverse congruence on $S$. 
We find necessary and sufficient conditions different from those of Theorem 3.8 for an $E^{*}$-dense semigroup $S$ to have a (Brandt) primitive inverse congruence. In fact, we show that $S$ has such a congruence if and only if it is (strongly) categorical and $D(S)$ is strongly *-dense in $S$, where $D(S)$ is the least full, weakly self-conjugate subsemigroup of $S$. We say that an $E^{*}$-dense semigroup $S$ is strongly $E^{*}$-dense if $D(S)$ is a strongly *dense subsemigroup of $S$. As noted above, a strongly $E^{*}$-dense semigroup has a minimum proper primitive inverse congruence, and, as an analogue of [18, Proposition 9], we give an explicit description of this congruence.

A weak inverse of an element $a$ of a semigroup is an element $b$ such that $b a b=b$. The set of all weak inverses of $a$ is denoted by $W(a)$, and, if $a$ is non-zero, then $W^{*}(a)$ is the set of all non-zero weak inverses of $a$.

Lemma 4.1. Let $S$ be a semigroup with zero, $P$ be a primitive inverse semigroup, and $\alpha: S \rightarrow P$ be a proper morphism. If $a$ is a non-zero element of $S$ and $a^{\prime} \in W^{*}(a)$, then $a^{\prime} \alpha$ is the inverse of $a \alpha$ in $P$.

Proof. Both $a \alpha$ and $a^{\prime} \alpha$ are non-zero, and, since $a^{\prime} a a^{\prime}=a^{\prime}$, it follows that $(a \alpha)\left(a^{\prime} \alpha\right)=$ $\left(a a^{\prime}\right) \alpha$ is a non-zero idempotent of $P$. Similarly, $\left(a^{\prime} \alpha\right)(a \alpha) \in E^{*}(P)$. Since $P$ is primitive inverse, it follows that $a \alpha \mathcal{L}\left(a^{\prime} \alpha\right)(a \alpha)$, and, hence, that $a^{\prime} \alpha=(a \alpha)^{-1}$.

We quote the following result from [6]. Its easy proof can be extracted from [10] and it summarizes some elementary properties of $E^{*}$-dense semigroups.

Proposition 4.2. Let $S$ be a semigroup with zero and $E=E(S)$. Then the following conditions are equivalent:

(1) $S$ is $E^{*}$-dense;

(2) for every non-zero element $a$ of $S$, there is an element $b$ of $S$ such that $a b \in E^{*}$ and $b a \in E^{*}$;

(3) for every non-zero element $a$ of $S$, there is an element $c$ of $S$ such that ac $\in E^{*}$;

(4) for every non-zero element a of $S$, there is an element $d$ of $S$ such that $d a \in E^{*}$; and

(5) every non-zero element of $S$ has a non-zero weak inverse.

A subsemigroup $T$ of $S$ is said to be closed under weak conjugation or weakly selfconjugate if $a t a^{\prime}, a^{\prime} t a \in T$ for all $t \in T, a \in S$ and $a^{\prime} \in W(a)$. Clearly, in a semigroup with zero, the intersection of a family of weakly self-conjugate subsemigroups contains zero and is weakly self-conjugate. Hence, we can define $D(S)$ to be the least (under inclusion) full, weakly self-conjugate subsemigroup of $S$.

There is a well-known construction of $D(S)$ [1], which we now describe. First, for any subsemigroup $T$ of $S$, put

$$
q(T)=\left\{a t a^{\prime}, a^{\prime} t a \mid t \in T, a, a^{\prime} \in S^{1}, a^{\prime} a a^{\prime}=a^{\prime}\right\}
$$


and $Q(T)=\langle q(T)\rangle$. Now put $D_{0}(S)=\langle E(S)\rangle$, and, for each non-negative integer $i$, put $D_{i+1}=Q\left(D_{i}(S)\right)$. Clearly, we have an ascending chain

$$
D_{0}(S) \subseteq D_{1}(S) \subseteq \cdots \subseteq D_{i}(S) \subseteq \cdots,
$$

and $D^{\prime}=\bigcup_{i \geqslant 0} D_{i}(S)$ is a subsemigroup of $S$. In fact, it is not difficult to see that $D^{\prime}=$ $D(S)$.

Lemma 4.3. Let $S$ be a categorical semigroup, $P$ be a primitive inverse semigroup and let $\alpha: S \rightarrow P$ be a proper surjective morphism. Then $D(S) \alpha \subseteq E(P)$.

Proof. Clearly, $D_{0}(S) \alpha=\langle E(S)\rangle \alpha \subseteq E(P)$. Suppose that $D_{i}(S) \alpha \subseteq E(P)$ and that $a \in S^{1}, t \in D_{i}(S)$ and $a^{\prime} \in W(a)$. Then either $a t a^{\prime}=0$, in which case $\left(a t a^{\prime}\right) \alpha \in E(P)$, or $a^{\prime} \in W^{*}(a)$. If $a=1$, then $a^{\prime}$ is idempotent so that $t a^{\prime} \in D_{i}(S)$ and $\left(a t a^{\prime}\right) \alpha \in$ $E(P)$. Otherwise, by Lemma 4.1, $a^{\prime} \alpha=(a \alpha)^{-1}$. Hence, $\left(a t a^{\prime}\right) \alpha=(a \alpha)(t \alpha)(a \alpha)^{-1}$, which belongs to $E(P)$ since $t \alpha \in E(P)$ by assumption and $P$ is inverse. Similarly, $\left(a^{\prime} t a\right) \alpha \in$ $E(P)$, and so $q\left(D_{i}(S)\right) \alpha \subseteq E(P)$. Hence, $D_{i+1}(S) \alpha \subseteq E(P)$ and the lemma follows by induction.

We now define a subsemigroup $T$ of a categorical $E^{*}$-dense semigroup $S$ to be weakly *-self-conjugate if it contains 0 , and, for all non-zero elements $a$ of $S$ and all $a^{\prime} \in W^{*}(a)$ and $t \in T^{1}$, if one of at or $t a^{\prime}$ is non-zero, then $a t a^{\prime} \in T^{*}$, and if one of $t a$ or $a^{\prime} t$ is non-zero, then $a^{\prime} t a \in T^{*}$.

It is clear that if $T$ is weakly *-self-conjugate, then it is weakly self-conjugate. It is also clear that if, in addition, $T$ contains no non-zero nilpotents, then $T$ is strongly *dense. The converse of the last assertion, when $T$ is full and weakly self-conjugate, is a consequence of the following proposition.

Proposition 4.4. Let $S$ be a categorical $E^{*}$-dense semigroup, $P$ be a primitive inverse semigroup, and $\alpha: S \rightarrow P$ be a proper surjective morphism. If $T$ is a non-zero weakly self-conjugate full subsemigroup of $S$ such that $T \alpha \subseteq E(P)$, then $T$ has no non-zero nilpotents and is weakly *-self-conjugate.

Proof. Since $\alpha$ is proper, we have $T^{*} \alpha \subseteq E^{*}(P)$ and, hence, if $t \in T^{*}$, then $t^{2} \neq 0$ and so $T$ has no non-zero nilpotents.

Let $a$ be a non-zero element of $S$ and $a^{\prime} \in W^{*}(a)$. Then, certainly, $a a^{\prime} \neq 0$. Suppose that $t \in T$ and $a t \neq 0$. Then $(a t) \alpha$ is non-zero, $t \alpha \in E^{*}(P)$, and, by Lemma 4.1, $a^{\prime} \alpha=(a \alpha)^{-1}$, so that $\left(t a^{\prime}\right) \alpha=(t \alpha)(a \alpha)^{-1}=((a \alpha)(t \alpha))^{-1}$ is non-zero. Hence $t a^{\prime} \neq 0$, and, by categoricity, $a t a^{\prime} \neq 0$. But $a t a^{\prime} \in T$ since $T$ is weakly self-conjugate and so ata $a^{\prime} \in T^{*}$.

Similar arguments give the other three conditions for $T$ to be weakly $*$-self-conjugate.

Corollary 4.5. Let $T$ be a weakly self-conjugate full subsemigroup of a categorical $E^{*}$-dense semigroup $S$. Then $T$ is strongly *-dense if and only if it has no non-zero nilpotents and is weakly *-self-conjugate. 
Proof. If $T$ is strongly *-dense, then, by definition, $T$ has no non-zero nilpotents and, by Proposition 3.1, $\rho_{T}$ is a proper primitive inverse congruence on $S$ with $T \subseteq \operatorname{Ker} \rho_{T}$. Hence, by Proposition 4.4, $T$ is weakly $*$-self-conjugate.

The converse is clear.

Corollary 4.6. Let $S$ be a categorical $E^{*}$-dense semigroup and suppose that $S$ has a proper primitive inverse congruence $\rho$. Then $D(S)$ is strongly *-dense.

Proof. By Lemma 4.3, $D(S) \subseteq \operatorname{Ker} \rho$ and, since $\rho$ is proper, $t \rho \in E^{*}(S / \rho)$ for any nonzero element $t$ of $D(S)$. The result now follows from Proposition 4.4 and Corollary 4.5 .

In general, if $S$ is a categorical $E^{*}$-dense semigroup, then $D(S)$ need not be strongly *-dense. For instance, it is easy to produce examples of idempotent generated completely 0 -simple semigroups (which are categorical) that contain non-zero nilpotent elements. For such a semigroup $S$ we have $D(S)=S$, so that $D(S)$ is not strongly *-dense.

On the other hand, if $S$ is $E^{*}$-dense and the idempotents of $S$ form a subsemigroup, then $D(S)$ is strongly *-dense. To prove this we use the following result.

Proposition 4.7. If $S$ is an $E$-dense semigroup in which $E(S)$ is a subsemigroup, then $E(S)=D(S)$.

Proof. The result follows from [5, Proposition 2.1], but, for completeness, we include a short proof from [7, Lemma 8.14]. Let $a \in S, a^{\prime} \in W(a)$ and $e \in E(S)$. Then $a^{\prime} a a^{\prime}=a^{\prime}$ and $a^{\prime} a \in E(S)$ so that

$$
\left(a e a^{\prime}\right)^{2}=a e a^{\prime} a e a^{\prime}=a e a^{\prime} a e a^{\prime} a a^{\prime}=a\left(e a^{\prime} a\right)^{2} a^{\prime}=a\left(e a^{\prime} a\right) a^{\prime}=a e a^{\prime} .
$$

Thus $a e a^{\prime} \in E(S)$. Similarly, $a^{\prime} e a \in E(S)$, and so $E(S)$ is weakly self-conjugate. Hence $E(S)=D(S)$.

Proposition 4.8. Let $S$ be a categorical $E^{*}$-dense semigroup such that $E(S)$ is a subsemigroup. Then $E(S)=D(S)$ and $E(S)$ is strongly *-dense.

Proof. Certainly, $S$ is $E$-dense and so, by Proposition 4.7, $E(S)=D(S)$.

It is clear that $E(S)$ contains no non-zero nilpotent elements. Further, by Proposition $4.2, W^{*}(a)$ is non-empty for every non-zero element $a$ of $S$. It follows from this and $[10$, Lemma 1.6$]$ that $E(S)$ is strongly $*$-dense.

We now give an analogue of [18, Proposition 9].

Theorem 4.9. Let $S$ be a categorical $E^{*}$-dense semigroup. Then $S$ has a proper primitive inverse congruence if and only if $D(S)$ is strongly *-dense. Moreover, if $D(S)$ is strongly *-dense, then the congruence $\rho_{D(S)}$ is the minimum proper primitive inverse congruence on $S$. 
Proof. If $S$ has a proper primitive inverse congruence, then, by Corollary 4.6, $D(S)$ is strongly $*$-dense.

Conversely, if $D(S)$ is strongly *-dense, then, by Proposition 3.1, the congruence $\rho_{D(S)}$ is proper and primitive inverse.

Now suppose that $D(S)$ is strongly *-dense and let $\tau$ be a proper primitive inverse congruence on $S$. Let $K=\operatorname{Ker} \tau$. Clearly, $E(S) \subseteq K$. Suppose that $k \in K^{1}$ and $a \in S$. Let $a^{\prime} \in W(a)$. If $k=1$, then $a k a^{\prime}$ and $a^{\prime} k a$ are idempotents and so belong to $K$. Otherwise, when $a k a^{\prime} \neq 0$, we have $\left(a k a^{\prime}\right) \tau=(a \tau)(k \tau)(a \tau)^{-1}$ by Lemma 4.1. Now, $(a \tau)(k \tau)(a \tau)^{-1}$ is idempotent and, hence, $a k a^{\prime} \in K$. Similarly, $a^{\prime} k a \in K$ and $K$ is weakly self-conjugate. Thus, $D(S) \subseteq K$ and so $\rho_{D(S)} \subseteq \rho_{K}$. But $\rho_{K}=\tau$ by Proposition 3.2 and, consequently, $\rho_{D(S)}$ is the minimum primitive inverse congruence on $S$.

Of course, if we suppose that $S$ is strongly categorical, then we can replace 'primitive inverse' by 'Brandt' in the above theorem.

The following corollary strengthens $[\mathbf{1 0}$, Theorem 2.1].

Corollary 4.10. Let $S$ be a (strongly) categorical $E^{*}$-dense semigroup whose idempotents form a subsemigroup. Then $S$ has a proper (Brandt) primitive inverse congruence and the relation

$$
\beta=\{(a, b) \mid a=b=0 \text { or } e a=b f \neq 0 \text { for some } e, f \in E(S)\}
$$

is the minimum proper primitive inverse (Brandt) congruence on $S$.

Proof. By Proposition 4.8, $E(S)=D(S)$ and, hence, the result follows from the theorem.

When $E(S)$ is actually a semilattice, we can recover the following result of Fountain and Hayes [6, Theorem 2.2], which generalizes [19, Theorem 2.7] and [22, Theorem 5].

Corollary 4.11. Let $S$ be a (strongly) categorical $E^{*}$-dense semigroup with $E(S)$ a semilattice. Then the relation

$$
\beta=\{(a, b) \mid a=b=0 \text { or } e a=e b \neq 0 \text { for some } e \in E(S)\}
$$

is the minimum primitive inverse (Brandt) congruence on $S$.

Proof. For the moment, denote the congruence of Corollary 4.10 by $\beta^{\prime}$. By Corollary 4.10 , it suffices to prove that $\beta^{\prime}=\beta$. Let $a, b \in S$ be such that $e a=b f \neq 0$ for some $e, f \in E$. Then eaf $=e b f \neq 0$. By [6, Lemma 2.1], it follows that there is an idempotent $k$ such that $k(e a)=k(e b) \neq 0$, that is, $(k e) a=(k e) b \neq 0$ and $(a, b) \in \beta$. Thus $\beta^{\prime} \subseteq \beta$.

Now, suppose that $e a=e b \neq 0$ and let $a^{\prime} \in W^{*}(a)$ and $b^{\prime} \in W^{*}(b)$. Then, by [10, Lemma 1.6], $f=a^{\prime} e a \in E^{*}(S)$, and, since $b f \neq 0$ follows from $a f \neq 0$, we also have $b f b^{\prime} \in E^{*}(S)$. Put $h=f b^{\prime} b$. Then

$$
a h=a f b^{\prime} b=a a^{\prime} e a b^{\prime} b=a a^{\prime} e^{2} a b^{\prime} b=e a a^{\prime} e a b^{\prime} b=e a f b^{\prime} b=e b f b^{\prime} b \neq 0
$$

and $e b f b^{\prime}$ is idempotent so that $(a, b) \in \beta^{\prime}$ and the corollary follows. 
We conclude this section with the following result for categorical $E^{*}$-dense semigroups.

Proposition 4.12. Let $S$ be a categorical $E^{*}$-dense semigroup and let $T$ be a weakly *-self-conjugate full subsemigroup of $S$. If $T$ is *-unitary, then it is also *-reflexive.

Proof. Suppose that $a, b \in S$ and $a b \in T^{*}$. By Proposition 4.2, $a$ has a non-zero weak inverse $a^{\prime}$. Also, $a^{\prime}(a b) \neq 0$ and, since $T$ is weakly $*$-self-conjugate, we have $a^{\prime} a b a \in T^{*}$. But $a^{\prime} a$ is idempotent and hence belongs to $T$, and so $b a \in T^{*}$ since $T$ is $*$-unitary. Thus, $T$ is *-reflexive as claimed.

\section{Proper primitive congruences on inverse semigroups}

We can, of course, specialize the results of $\S 3$ to obtain a characterization of primitive inverse congruences on categorical inverse semigroups. However, there is also a wellknown theory of congruences on arbitrary inverse semigroups (see [20, ch. III]) in which congruences are characterized by congruence pairs.

Recall that a subsemigroup $K$ of an inverse semigroup $S$ is self-conjugate if $a^{-1} K a \subseteq K$ for all $a \in S$ and that $K$ is a normal subsemigroup of $S$ if it is a full, self-conjugate, inverse subsemigroup. A congruence $\tau$ on $E(S)$ is normal if, for any $e, f \in E(S)$ and $a \in S$, we have $\left(a^{-1} e a, a^{-1} f a\right) \in \tau$ whenever $(e, f) \in \tau$.

The pair $(K, \tau)$ is a congruence pair for $S$ if $K$ is a normal subsemigroup of $S, \tau$ is a normal congruence on $E(S)$ and the following two conditions are satisfied for all $a \in S$, $e \in E(S), k \in K$ :

(i) if $a e \in K$ and $\left(e, a^{-1} a\right) \in \tau$, then $a \in K$; and

(ii) $\left(k k^{-1}, k^{-1} k\right) \in \tau$.

Given a congruence pair $(K, \tau)$ on $S$, the relation $\rho_{(K, \tau)}$ defined by

$$
a \rho_{(K, \tau)} b, \text { if and only if } a^{-1} a \tau b^{-1} b \text { and } a b^{-1} \in K
$$

is a congruence on $S$ with kernel $K$ and which restricts to $\tau$ on $E(S)$.

Our aim in this section is to show the connections between the kernel-trace approach and that of $\S 3$ to primitive inverse congruences on inverse semigroups.

We start by pointing out that a *-dense, *-unitary subsemigroup $T$ of any semigroup $S$ with zero is full. If $e \in E^{*}(S)$, then $e b \in T^{*}$ for some $b \in S$. But $e(e b)=e b$ and $T$ is *-unitary so that $e \in T$. On the other hand, if $S$ is inverse, then it is clear that any full subsemigroup is $*$-dense. Hence, the kernels of primitive inverse congruences on an inverse semigroup are the full, *-unitary, *-reflexive subsemigroups. We also point out that an easy consequence of a subsemigroup of an inverse semigroup being full and *-unitary is that it is an inverse subsemigroup.

Now let $T$ be a subsemigroup of an inverse semigroup with zero. We say that $T$ is *-self-conjugate when for all $a \in S$ and $t \in T^{*}$, if $t a^{-1} \neq 0$, then $a t a^{-1} \in T^{*}$. The next proposition relates this concept to others we have introduced earlier. 
Proposition 5.1. Let $T$ be a full subsemigroup of a categorical inverse semigroup $S$. Then the following conditions are equivalent:

(1) $T$ is *-self-conjugate;

(2) $T$ is weakly *-self-conjugate;

(3) $T$ is weakly self-conjugate and contains no non-zero nilpotents;

(4) $T$ is self-conjugate and contains no non-zero nilpotents; and

(5) $T$ is strongly *-dense.

Proof. If (1) holds, let $t \in T^{*}$. Then $t t^{-1} \neq 0$ so that $t^{2} t^{-1} \neq 0$ since $T$ is *-selfconjugate. Hence $t t^{-1} t^{-1} t=t^{-1} t t t^{-1} \neq 0$ by categoricity, and, hence, if $a \in S$ and at $\neq 0$, then $a t t^{-1} t^{-1} t \neq 0$. By [10, Lemma 1.6], att $t^{-1} t^{-1} t a^{\prime} \neq 0$ for any $a^{\prime} \in W^{*}(a)$. Hence $t a^{\prime} \neq 0$, and so $a t a^{\prime} \neq 0$ by categoricity. Now,

$$
a t a^{\prime}=a t a^{\prime} a a^{\prime}=a t a^{\prime} a a^{-1} a a^{\prime}=\left(a\left(t a^{\prime} a\right) a^{-1}\right) a a^{\prime}
$$

which is in $T$ since $T$ is full and *-self-conjugate.

Similar arguments give the other three conditions needed for weak $*$-self-conjugacy.

If (2) holds, then, clearly, $T$ is weakly self-conjugate. Also, if $t \in T^{*}$, then $t t^{-1} \neq 0$ and $t^{-1} \in W^{*}(t)$ so that $t^{2} t^{-1} \neq 0$ since $T$ is weakly $*$-self-conjugate. In particular, $t^{2} \neq 0$ and so $T$ contains no non-zero nilpotents.

That (3) implies (4) is immediate.

Suppose that (4) holds and let $a \in S, t \in T$ be such that $t a^{-1} \neq 0$. Then $a t^{-1}=$ $\left(t a^{-1}\right)^{-1} \neq 0$ and $t^{2} \neq 0$, so that

$$
a t t^{-1} t^{-1} t=a t^{-1} t t t^{-1} \neq 0
$$

and, in particular, $a t \neq 0$. Hence $a t a^{-1} \neq 0$ and $T$ is $*$-self-conjugate, that is, (1) holds.

Thus (1)-(4) are equivalent and it is now immediate from Corollary 4.5 that (5) is equivalent to these conditions.

If $T$ is a full, inverse subsemigroup of a categorical inverse semigroup and $T$ is *-selfconjugate, then $T$ is said to be $*$-normal. As an immediate consequence of Proposition 5.1 we have the following result.

Corollary 5.2. A subsemigroup of a categorical inverse semigroup is *-normal if and only if it is normal and contains no non-zero nilpotent elements.

Next we note a connection between $*$-self-conjugacy and $*$-reflexivity.

Lemma 5.3. If $T$ is a full, *-unitary subsemigroup of a categorical, inverse semigroup $S$, then $T$ is *-reflexive if and only if it is *-self-conjugate. 
Proof. If $T$ is *-self-conjugate, then, by Proposition 5.1 , it is weakly *-self-conjugate. Hence, by Proposition 4.12 , it is $*$-reflexive.

Conversely, if $T$ is $*$-reflexive and $t \in T^{*}, a \in S$ are such that $t a^{-1} \neq 0$, then $t a^{-1} a \neq 0$. Now $t a^{-1} a \in T$ since $T$ is full, and, hence, $a t a^{-1} \in T^{*}$, as required.

The following alternative characterization of kernels of primitive inverse congruences on categorical inverse semigroups now follows from Theorem 3.4 and the results of this section.

Proposition 5.4. A subsemigroup of a categorical inverse semigroup is the kernel of a primitive inverse congruence if and only if it is a *-unitary, *-normal subsemigroup.

Let $S$ be a categorical inverse semigroup. We define a relation $\tau$ on $E(S)$ by the rule that

$$
(e, f) \in \tau \text { if and only if } e=f=0 \text { or } e f \neq 0 \text {. }
$$

Proposition 5.5. If $T$ is a *-unitary, *-normal subsemigroup of $S$, then the pair $(T, \tau)$ is a congruence pair for $S$ and $\rho_{T}=\rho_{(T, \tau)}$.

Proof. It is easy to see that $\tau$ is a congruence on $E(S)$ in view of the fact that $S$ is categorical. If $e, f \in E(S)$ and $e f \neq 0$, then, for any $a \in S$ such that $a e \neq 0$, we have

$$
a e a^{-1} a f a^{-1}=a a^{-1} a e f a^{-1}=a e f a^{-1} \neq 0
$$

by categoricity, and, hence, $\tau$ is a normal congruence on $E(S)$.

Let $a \in S$ and $e \in E(S)$ with $a e \in T$ and $\left(e, a^{-1} a\right) \in \tau$. If $e=0$, then $a=0$ and $a \in T$. If $e \neq 0$, then $a^{-1}$ ae $\neq 0$, and, since $T$ is $*$-unitary, it follows that $a \in T$.

If $t \in T^{*}$, then $t^{2} \neq 0$, and it follows easily that $t^{-1} t \tau t t^{-1}$. Thus $(T, \tau)$ is a congruence pair.

If $a \rho_{T} b$ and $a \neq 0$, then $x a=b t \neq 0$ for some $x, t \in T$. Hence $x a b^{-1}=b t b^{-1}$, and, since $T$ is *-normal, $x a b^{-1} \in T$. As $x \in T$ and $T$ is also $*$-unitary, we have $a b^{-1} \in T$. Now $a^{-1} a b^{-1} b \neq 0$, and so $(a, b) \in \rho_{(T, \tau)}$.

Conversely, if $(a, b) \in \rho_{(T, \tau)}$ and $a \neq b$, then $a^{-1} a \tau b^{-1} b$ and $a b^{-1} \in T$. Hence $a^{-1} a b^{-1} b \neq 0$, so that, in particular, $a b^{-1} b \neq 0$. It follows that $a \rho_{T} b$ since $b^{-1} b$ and $a b^{-1}$ are both in $T$.

Thus $\rho_{T}=\rho_{(T, \tau)}$.

Corollary 5.6. If $T$ is a *-unitary, *-normal subsemigroup of a categorical inverse semigroup $S$, then, for all $a, b \in S$,

$$
(a, b) \in \rho_{T} \text { if and only if } a=b=0 \text { or } a b^{-1} \in T^{*} .
$$

Proof. If $(a, b) \in \rho_{T}$, then $(a, b) \in \rho_{(T, r)}$, so that, if $a \neq 0$, then $a b^{-1} \in T^{*}$.

Conversely, if $a b^{-1} \in T^{*}$, then $a b^{-1} b \neq 0$, and, hence, $(a, b) \in \rho_{T}$. 


\section{Joins with primitive inverse congruences}

Recently, Edwards [4] proved that given a group congruence $\gamma$ and an arbitrary congruence $\rho$ on a semigroup, the join of $\gamma$ with $\rho$ is exactly $\gamma \circ \rho \circ \gamma$. As a consequence, any group congruence $\gamma$ on a semigroup $S$ is a dually right modular element of the lattice of all congruences on $S$ in the sense of Jones [13].

In this section we show that very similar arguments yield a similar result for proper primitive inverse congruences on categorical semigroups. As in the case of group congruences, this result can be used to give an explicit description of the join of a primitive inverse congruence and an arbitrary proper congruence and a description of the kernel of such a join.

Proposition 6.1. Let $\gamma$ and $\rho$ be proper congruences on a categorical semigroup $S$. If $\gamma$ is primitive inverse, then $\gamma \vee \rho=\gamma \circ \rho \circ \gamma$.

Proof. We modify the proof of $[4$, Theorem 1]. As there, we need only show that the relation $\gamma \circ \rho \circ \gamma$ is transitive.

Let $a, b, c$ be non-zero elements of $S$ with $(a, b),(b, c) \in \gamma \circ \rho \circ \gamma$. Then there are elements $x, y, u, v$ of $S$ such that $a \gamma x \rho y \gamma b$ and $b \gamma u \rho v \gamma c$. Now, $y \gamma=b \gamma=u \gamma$ in the primitive inverse semigroup $S / \gamma$ so that if $s \in S$ is such that $s \gamma=(y \gamma)^{-1}$, then $(u s) \gamma$ and $(s y) \gamma$ are non-zero idempotents of $S / \gamma$. By categoricity, $u s y \neq 0$ and, as $(u s y, u s x) \in \rho$, we have $u s x \neq 0$. It now follows from Lemma 2.6 that $u s x \gamma x$.

Similarly, vsy $\gamma v$ and, hence, $(a, u s x) \in \gamma,(u s x, v s y) \in \rho$ and $(v s y, c) \in \gamma$ so that $(a, c) \in \gamma \circ \rho \circ \gamma$.

An example is given in [4] to show that, in general, $\rho \vee \theta \neq \rho \circ \theta \circ \rho$ for arbitrary congruences $\rho, \theta$ on a semigroup $S$. By adjoining a zero to $S$, we see that this is also the case for proper congruences on a categorical semigroup.

The following lemma is another illustration of the similarity between groups and primitive inverse semigroups.

Lemma 6.2. If $\rho, \sigma$ are proper congruences on a primitive inverse semigroup $S$, then $\rho \circ \sigma=\sigma \circ \rho$.

Proof. Since ef $=0$ for distinct idempotents $e, f$ of $S$, it follows that any proper congruence is idempotent separating. The lemma now follows from [2, Theorem 7.56].

In fact, on a Brandt semigroup the only congruence that is not proper is the universal congruence [21]. It follows easily from this and the lemma that any two congruences on a primitive inverse semigroup commute.

Corollary 6.3. Let $\rho$ and $\sigma$ be proper primitive inverse congruences on a categorical semigroup $S$. Then $\rho \vee \sigma=\rho \circ \sigma \circ \rho=\sigma \circ \rho \circ \sigma$.

Moreover, if $\rho \cap \sigma$ is a primitive inverse congruence on $S$, then $\rho \vee \sigma=\rho \circ \sigma=\sigma \circ \rho$.

Proof. The first part is immediate by Proposition 6.1. Let $\beta=\rho \cap \sigma$ and suppose that $\beta$ is primitive inverse. Then $\rho / \beta$ and $\sigma / \beta$ are (proper) congruences on the primitive 
inverse semigroup $S / \beta$ so that, by Lemma 6.2 , they commute. Hence $\rho$ and $\sigma$ commute.

Proposition 6.4. Let $\gamma$ be a primitive inverse congruence and $\rho$ be an arbitrary proper congruence on a categorical semigroup $S$. Then, for all $a, b \in S,(a, b) \in \gamma \vee \rho$ if and only if $a=b=0$ or $x a \rho b t$ for some $x, t \in \operatorname{Ker} \gamma$ with $x a \neq 0$.

Proof. The proof is a simple modification of that of Theorem 6 of [4]. Let $K=\operatorname{Ker} \gamma$ and let $a, b$ be non-zero elements of $S$ such that $(a, b) \in \gamma \vee \rho$. Then, by Theorem 6.1, there are elements $c, d$ of $S$ such that $a \gamma c \rho d \gamma b$. By Proposition 3.2, there are elements $x, t, h, k$ of $K$ such that

$$
x a=c t \neq 0 \quad \text { and } \quad h d=b k \neq 0 .
$$

As $\gamma$ and $\rho$ are proper and $x a \neq 0$, we have $x b \neq 0$ so that $x h d=x b k \neq 0$ and, in particular, $x h \neq 0$. Hence $x h \in K^{*}$, and so $h x \in K^{*}$ since $K$ is $*$-reflexive. Now, $(h c t, h d t) \in \rho$ and $h x a=h c t, h d t=b k t$ so that $(h x a, b k t) \in \rho$. Also, $h x, k t \in K$ and $h x a \neq 0$, as required.

Conversely, suppose that $a, b$ are non-zero elements of $S$ and that $x a \rho b t$ for some $x, t \in K$ with $x a \neq 0$. Since $x, t \in K$ and $S / \gamma$ is primitive, it follows from Lemma 2.6 that $x a \gamma a$ and $b t \gamma b$. Thus $(a, b) \in \gamma \vee \rho$, as required.

Finally, we give an analogue of [4, Theorem 7]. First, we define the $*$-closure of a subset $A$ of a semigroup $S$ with zero to be the subset $A \omega^{*}$, where

$$
A \omega^{*}=\left\{x \in S \mid a x \in A^{*} \text { for some } a \in A\right\} .
$$

Proposition 6.5. If $\gamma$ is a primitive inverse congruence and $\rho$ is any proper congruence on a categorical semigroup $S$, then $\operatorname{Ker}(\gamma \vee \rho)=((\operatorname{Ker} \gamma) \rho) \omega^{*} \cup\{0\}$.

Proof. Let $K=\operatorname{Ker}(\gamma \vee \rho)$. Now $\gamma \vee \rho$ is proper so that if $a \in K^{*}$, then $a^{2} \in K^{*}$. By Proposition 6.4, $x a^{2} \rho a t$ for some $x, t \in \operatorname{Ker} \gamma$ with $x a \neq 0$. Now $\operatorname{Ker} \gamma$ is strongly *-dense and so there is an element $z$ of $S$ such that $a z, z a$ are non-zero and in $\operatorname{Ker} \gamma$. By categoricity, $x a z \neq 0$. Also, $x a z \in \operatorname{Ker} \gamma$ so that $z x a$ is a non-zero element of $\operatorname{Ker} \gamma$ by $*$-reflexivity. Furthermore, $z x a^{2} \rho z a t$ and $z a t \in \operatorname{Ker} \gamma$ is non-zero, and, hence, $a \in$ $((\operatorname{Ker} \gamma) \rho) \omega^{*}$.

Conversely, if $a \in((\operatorname{Ker} \gamma) \rho) \omega^{*}$, then there is an element $h$ of $(\operatorname{Ker} \gamma) \rho$ such that $h a \in(\operatorname{Ker} \gamma) \rho$ and $h a \neq 0$. Let $p, q \in \operatorname{Ker} \gamma$ be such that $h \rho p$ and $h a \rho q$. Now $(h a)^{2} \rho q^{2} \gamma q$ and $q \neq 0$ since $h a \neq 0$. Hence $(h a)^{2} \neq 0$, and, since $(p a)^{2} \rho(h a)^{2}$, we have $(p a)^{2} \neq 0$. However, $(p a)^{2} \gamma a^{2}$ since $p \in \operatorname{Ker} \gamma$, and, consequently, $\left(a^{2}, h a\right) \in \gamma \vee \rho$. Since happa and pa $\gamma a$, we obtain $\left(a^{2}, a\right) \in \gamma \vee \rho$. Thus $a \in K$.

Acknowledgements. Part of this research was done while the first author was visiting the mathematics department of Wilfrid Laurier University. He expresses his thanks to the department and particularly to Syd Bulman-Fleming for the friendly atmosphere and kind hospitality provided. He also acknowledges support from project FACC 9/98/1/ 0023 of FCT, Portugal. 
The work of the second author was partly supported by the INVOTAN grant 4/c/96/ $\mathrm{PO}$ and the research was carried out as part of the project Praxis 2/2.1/MAT/73.94.

We thank the referee for drawing our attention to $[\mathbf{1 7}]$ and suggesting Theorem 3.8 .

\section{References}

1. J.-C. Birget, S. MARgolis and J. Rhodes, Semigroups whose idempotents form a subsemigroup, Bull. Aust. Math. Soc. 41 (1990), 161-184.

2. A. H. Clifford And G. B. Preston, The algebraic theory of semigroups, vol. II (American Mathematical Society, Providence, RI, 1967).

3. P. Dubreil, Contribution à la théorie des semigroupes, Mém. Acad. Sci. Inst. France 63 (1941), 52.

4. P. M. Edwards, On joins with group congruences, Proc. Edinb. Math. Soc. 40 (1997), 63-67.

5. J. Fountain, $\langle E\rangle$-dense monoids, in Monash conference on semigroup theory (ed. T. E. Hall, P. R. Jones and J. C. Meakin), pp. 68-76 (World Scientific, Singapore, 1991).

6. J. Fountain AND A. D. HAYES, $E^{*}$-dense semigroups whose idempotents form a subsemigroup, Preprint, University of York.

7. J. Fountain, J.-E. PIN and P. Weil, Covers for monoids, Preprint, University of York.

8. G. M. S. GOMES, A characterisation of the group congruences on a semigroup, Semigroup Forum 46 (1993), 48-53.

9. G. M. S. Gomes AND J. M. HowIE, A $P$-theorem for inverse semigroups with zero, Portugaliae Math. 53 (1996), 257-278.

10. G. M. S. Gomes ANd J. M. HowiE, Semigroups with zero whose idempotents form a subsemigroup, Proc. R. Soc. Edinb. A 128 (1998), 265-281.

11. T. E. Hall AND W. D. MUnN, The hypercore of a semigroup, Proc. Edinb. Math. Soc. 28 (1985), 107-112.

12. J. M. HowIE, Fundamentals of semigroup theory (Oxford University Press, 1995).

13. P. R. JONES, Joins and meets of congruences on a regular semigroup, Semigroup Forum 30 (1984), 1-16.

14. G. Lallement and M. Petrich, Some results concerning completely 0-simple semigroups, Bull. Am. Math. Soc. 70 (1964), 777-778.

15. F. W. Levi, On semigroups, Bull. Calcutta Math. Soc. 36 (1944), 141-146.

16. F. W. Levi, On semigroups, II, Bull. Calcutta Math. Soc. 38 (1946), 123-124.

17. D. B. MCAlister, Matrix representations of semigroups, Glasgow Math. J. 8 (1967), 1-13.

18. H. Mitsch, Subdirect products of E-inversive semigroups, J. Aust. Math. Soc. 48 (1990), 66-78.

19. W. D. MUNn, Brandt congruences on inverse semigroups, Proc. Lond. Math. Soc. (3) 14 (1964), 154-164.

20. M. PETRICH, Inverse semigroups (Wiley, New York, 1984).

21. G. B. Preston, Congruences on Brandt semigroups, Math. Ann. 139 (1959), 91-94.

22. G. B. Preston, Matrix representations of inverse semigroups, J. Aust. Math. Soc. 9 (1969), 29-61. 